\title{
Platelet-Derived Growth Factor-AA
}

National Cancer Institute

\section{Source}

National Cancer Institute. Platelet-Derived Growth Factor-AA. NCI Thesaurus. Code C128285.

A disulfide-linked homodimeric protein complex comprised of 2 platelet-derived growth factor subunit A proteins. This dimeric growth factor binds to a homodimeric receptor comprised of two platelet-derived growth factor receptor alpha proteins. Ligand binding activates the intrinsic tyrosine-protein kinase activity of the receptor complex and initiates signal transduction pathways. 